Charles Swartz, Department of Mathematical Sciences, New Mexico State

University, Las Cruces, NM 88003, USA, e-mail:cswartz@nmsu.edu

\title{
UNIFORM INTEGRABILITY AND MEAN CONVERGENCE FOR THE VECTOR-VALUED MCSHANE INTEGRAL
}

\begin{abstract}
We show that a pointwise convergent, uniformly integrable sequence of Banach space valued, McShane integrable functions converges in mean. We also show that uniform integrability holds in a vector-valued generalization of the Beppo Levi convergence theorem.
\end{abstract}

It has been observed in $[3,4,5]$, [7] that uniform integrability for the Henstock-Kurzweil integral is a sufficient condition to "take the limit under the integral sign." In this note we point out that uniform integrability for the McShane integral is actually a sufficient condition for mean or $L^{1}$ convergence. Our methods extend easily to functions with values in a Banach space so we consider this case where the results give significant improvements to the scalar case. We also show that the conclusion of the vector-valued generalization of the Monotone Convergence (Beppo Levi) Theorem given in [10] can be improved to uniform integrability.

We fix the notation and terminology which will be used in the sequel. It should be noted that we will work in $\mathbb{R}$ whereas the results in $[3,4,5]$ are for compact intervals in $\mathbb{R}$. Let $X$ be a (real) Banach space and let $\mathbb{R}^{*}$ be the extended real line with the points $\pm \infty$ added. If $f$ is any function $f: \mathbb{R} \rightarrow X$, we always assume that $f$ is extended to $\mathbb{R}^{*}$ by setting $f( \pm \infty)=0$.

A gauge is a function $\gamma$ on $\mathbb{R}^{*}$ whose value at a point $t$ is a neighborhood $\gamma(t)$ of $t$, where $\gamma(t)$ is bounded whenever $t \in \mathbb{R}$. [A neighborhood of $\infty$ is an interval of the form $(a, \infty]$; similarly for $-\infty$.] A partition of $\mathbb{R}$ is a finite collection of left-closed intervals $\left\{I_{i}: i=1, \ldots, n\right\}$ such that $I_{i} \cap I_{j}=\phi$ for $i \neq j$ and $\mathbb{R}=\bigcup_{i=1}^{n} I_{i}$ (here we agree that $(-\infty, a)$ is left-closed). A tagged partition of $\mathbb{R}$ is a finite collection of pairs $\left\{\left(I_{i}, t_{i}\right): i=1, \ldots, n\right\}$ such that

Key Words: McShane integral, uniform integrability, mean convergence

Mathematical Reviews subject classification: 28B05

Received by the editors March 25, 1997 
$\left\{I_{i}: i=1, \ldots, n\right\}$ is a partition of $\mathbb{R}$ and $t_{i} \in \mathbb{R}^{*} ; t_{i}$ is called the tag associated with $I_{i}$. Note that it is not required that $t_{i} \in I_{i}$; this requirement is what distinguishes the McShane and the Henstock-Kurzweil integral ([1],[7],[8],[3]). If $\gamma$ is a gauge on $\mathbb{R}^{*}$, a tagged partition $\mathcal{D}=\left\{\left(I_{i}, t_{i}\right): i=1, \ldots, n\right\}$ is $\gamma$-fine if $\bar{I}_{i} \subset \gamma\left(t_{i}\right)$ for $i=1, \ldots, n$. If $\mathcal{D}=\left\{\left(I_{i}, t_{i}\right): i=1, \ldots, n\right\}$ is a tagged partition and $f: \mathbb{R} \rightarrow X$, we write $S(f, \mathcal{D})=\sum_{i=1}^{n} f\left(t_{i}\right) \ell\left(I_{i}\right)$ for the Riemann sum of $f$ with respect to $\mathcal{D}$ where $\ell$ is Lebesgue measure on $\mathbb{R}$ [here we make the usual agreement that $0 \cdot \infty=0$ ].

Definition 1. A function $f: \mathbb{R} \rightarrow X$ is (McShane) integrable over $\mathbb{R}$ if there exists $v \in X$ such that for every $\varepsilon>0$ there exists a gauge $\gamma$ on $\mathbb{R}^{*}$ such that $\|S(f, \mathcal{D})-v\|<\varepsilon$ whenever $\mathcal{D}$ is $\gamma$-fine.

The vector $v$ is called the (McShane) integral of $f$ over $\mathbb{R}$ and is denoted by $\int_{\mathbb{R}} f$. We refer the reader to [8], [3] for basic properties of the McShane integral.

Let $M(\mathbb{R}, X)$ be the space of all $X$-valued integrable functions defined on $\mathbb{R}$; if $X=\mathbb{R}$, we abbreviate $M(\mathbb{R}, \mathbb{R})=M(\mathbb{R})$. The space $M(\mathbb{R})$ is complete under the semi-norm $\|f\|_{1}=\int_{\mathbb{R}}|f|$ ([8, VI.4.3]). We define a seminorm on $M(\mathbb{R}, X)$ by $\|f\|_{1}=\sup \left\{\int_{\mathbb{R}}\left|x^{\prime} f\right|: x^{\prime} \in X^{\prime},\left\|x^{\prime}\right\| \leq 1\right\}$; in general, \|\|$_{1}$ is not complete ([10]; see [4], [2] for properties of the vector-valued McShane integral and its comparison with the Pettis and Bochner integrals). We describe another semi-norm which is equivalent to \|\|$_{1}$ and is useful in estimation. Let $\mathcal{A}$ be the algebra of subsets of $\mathbb{R}$ generated by the leftclosed subintervals of $\mathbb{R}$; thus, every element of $\mathcal{A}$ is a finite, pairwise disjoint union of left-closed intervals $([9,2.1 .11])$. If $f \in M(\mathbb{R}, X), A \in \mathcal{A}$ and $C_{A}$ denotes the characteristic function of $A$, then $C_{A} f$ is also integrable and $\|f\|_{1}^{\prime}=\sup \left\{\left\|\int_{\mathbb{R}} C_{A} f\right\|=\left\|\int_{A} f\right\|: A \in \mathcal{A}\right\}$ defines a semi-norm on $M(\mathbb{R}, X)$ which is equivalent to \|\|$_{1}([10])$.

We next define uniform (McShane) integrability and show that uniform integrability implies convergence in \|\|$_{1}$. A family $\mathcal{F}$ of $X$-valued functions defined on $\mathbb{R}$ is uniformly integrable if for every $\varepsilon>0$ there exists a gauge $\gamma$ on $\mathbb{R}^{*}$ such that $\left\|S(f, \mathcal{D})-\int_{\mathbb{R}} f\right\|<\varepsilon$ for every $f \in \mathcal{F}$ and $\mathcal{D} \gamma$-fine; that is, the gauge is independent of the functions in $\mathcal{F}$.

For our next theorems we require an important result called the Henstock Lemma. If $\mathcal{D}=\left\{\left(I_{i}, t_{i}\right): i=1, \ldots, n\right\}$ is any collection of pairwise disjoint, left-closed subintervals $\left\{I_{i}\right\}$ and $t_{i} \in \mathbb{R}^{*}$, then $\mathcal{D}$ is called a partial tagged partition of $\mathbb{R}$ (it is not required that $\bigcup_{i=1}^{n} I_{i}=\mathbb{R}$ ); if $\gamma$ is a gauge on $\mathbb{R}^{*}, \mathcal{D}$ is $\gamma$-fine if $\bar{I}_{i} \subset \gamma\left(t_{i}\right)$ for $i=1, \ldots, n$. We again write $S(f, \mathcal{D})=\sum_{i=1}^{n} f\left(t_{i}\right) \ell\left(I_{i}\right)$. 
Lemma 2. (Henstock) Let $f \in M(\mathbb{R}, X)$ and $\varepsilon>0$. Suppose the gauge $\gamma$ on $\mathbb{R}^{*}$ is such that $\left\|S(f, \mathcal{D})-\int_{\mathbb{R}} f\right\|<\varepsilon$ for every $\gamma$-fine tagged partition $\mathcal{D}$ of $\mathbb{R}$. If $\mathcal{D}$ is any $\gamma$-fine partial tagged partition and $I=\bigcup_{i=1}^{n} I_{i}$, then $\left\|S(f, \mathcal{D})-\int_{I} f\right\| \leq \varepsilon$.

See [4] for Lemma 2.

We first establish an interesting preliminary result.

Theorem 3. Let $f_{k} \in M(\mathbb{R}, X)$ for every $k \in \mathbb{N}$. If $\left\{f_{k}\right\}$ is uniformly integrable over $\mathbb{R}$, then

$$
\lim _{b \rightarrow \infty}\left\|C_{[b, \infty)} f_{k}\right\|_{1}^{\prime}=0
$$

uniformly for $k \in \mathbb{N}$.

Proof. Let $\varepsilon>0$. There exists a gauge $\gamma$ with $\gamma(z)$ bounded for every $z \in \mathbb{R}$ such that $\left\|\int_{\mathbb{R}} f_{k}-S\left(f_{k}, \mathcal{D}\right)\right\|<\varepsilon$ when $\mathcal{D}$ is $\gamma$-fine. Fix such a $\mathcal{D}=$ $\left\{\left(I_{i}, t_{i}\right): 1 \leq i \leq m\right\}$ and assume that $I_{1}=[b, \infty), t_{1}=\infty$. If $a \geq b$, let $A$ be an element of $\mathcal{A}$ with $A \subset[a, \infty)$ and $A=\bigcup_{i=1}^{n} J_{i}, J_{i}$ a left-closed interval and $\left\{J_{i}\right\}$ pairwise disjoint. Then $\mathcal{J}=\left\{\left(J_{i}, \infty\right): 1 \leq i \leq n\right\}$ is a $\gamma$-fine partial tagged partition so Henstock's Lemma implies that

$$
\left\|\int_{A} f_{k}-S\left(f_{k}, \mathcal{J}\right)\right\|=\left\|\int_{A} f_{k}\right\| \leq \varepsilon
$$

Since $A \in \mathcal{A}$ is arbitrary, $\left\|C_{[a, \infty)} f_{k}\right\|_{1}^{\prime} \leq \varepsilon$ for $a \geq b$.

We next establish our mean convergence result.

Theorem 4. Let $f_{k}: \mathbb{R} \rightarrow X$ be integrable for every $k \in \mathbb{N}$ and suppose $\left\{f_{k}\right\}$ converges pointwise to $f$. If $\left\{f_{k}\right\}$ is uniformly integrable, then $f$ is integrable and $\left\|f_{k}-f\right\|_{1} \rightarrow 0$.

Proof. Let $\varepsilon>0$. There exists a gauge $\gamma$ with $\gamma(z)$ bounded for every $z \in \mathbb{R}$ such that $\left\|\int_{\mathbb{R}} f_{k}-S\left(f_{k}, \mathcal{D}\right)\right\|<\varepsilon$ whenever $\mathcal{D}$ is $\gamma$-fine.

Fix a tagged partition $\mathcal{D}=\left\{\left(I_{i}, t_{i}\right): 1 \leq i \leq m\right\}$ which is $\gamma$-fine. Since $\left\{f_{k}\right\}$ is pointwise convergent and $f_{k}( \pm \infty)=0$, there exists $N$ such that $k, j \geq N$ implies $\left\|S\left(f_{k}, \mathcal{D}\right)-S\left(f_{j}, \mathcal{D}\right)\right\|=\left\|\sum_{i=1}^{m}\left(f_{k}\left(t_{i}\right)-f_{j}\left(t_{i}\right)\right) \ell\left(I_{i}\right)\right\|<\varepsilon$. Therefore, 
if $k, j \geq N$, we have

$$
\begin{aligned}
\left\|\int_{\mathbb{R}} f_{k}-\int_{\mathbb{R}} f_{j}\right\| \leq & \left\|\int_{\mathbb{R}} f_{k}-S\left(f_{k}, \mathcal{D}\right)\right\|+\left\|S\left(f_{k}, \mathcal{D}\right)-S\left(f_{j}, \mathcal{D}\right)\right\| \\
& +\left\|S\left(f_{j}, \mathcal{D}\right)-\int_{\mathbb{R}} f_{j}\right\|<3 \varepsilon .
\end{aligned}
$$

Hence, $\lim _{k} \int_{\mathbb{R}} f_{k}=L$ exists in $X$.

We claim that $\int_{\mathbb{R}} f=L$. Let $\mathcal{E}$ be $\gamma$-fine. Pick $n_{0}$ such that $k \geq n_{0}$ implies $\left\|\int_{\mathbb{R}} f_{k}-L\right\|<\varepsilon$. As above there exists $n_{1} \geq n_{0}$ such that whenever $k \geq n_{1}$, it follows that $\left\|S(f, \mathcal{E})-S\left(f_{k}, \mathcal{E}\right)\right\|<\varepsilon$. Then

$$
\begin{aligned}
\|L-S(f, \mathcal{E})\| \leq & \left\|L-\int_{\mathbb{R}} f_{n_{1}}\right\|+\left\|\int_{\mathbb{R}} f_{n_{1}}-S\left(f_{n_{1}}, \mathcal{E}\right)\right\| \\
& +\left\|S\left(f_{n_{1}}, \mathcal{E}\right)-S(f, \mathcal{E})\right\|<3 \varepsilon
\end{aligned}
$$

and the claim is established.

For the last statement we may assume that $f=0$ since $f_{k}-f \rightarrow 0$ pointwise and $\left\{f_{k}-f\right\}$ is uniformly integrable. With $\mathcal{D}$ fixed as above, assume that $I_{1}=[b, \infty)$ and $I_{2}=(-\infty, a)$ and set $I=\mathbb{R} \backslash I_{1} \cup I_{2}$. Let $A$ be an arbitrary element of $\mathcal{A}$ with $A=\bigcup_{j=1}^{n} J_{j}, J_{j}$ a left-closed interval, $\left\{J_{j}\right\}$ pairwise disjoint. Then $\mathcal{E}=\left\{\left(I_{i} \cap J_{j}, t_{i}\right): 1 \leq i \leq m, 1 \leq j \leq n\right\}$ is $\gamma$-fine so Henstock's Lemma implies $\left\|\sum_{i=1}^{m} \sum_{j=1}^{n}\left\{\int_{I_{i} \cap J_{j}} f_{k}-f_{k}\left(t_{i}\right) \ell\left(I_{i} \cap J_{j}\right)\right\}\right\| \leq \varepsilon$. Hence,

$$
\begin{aligned}
\left\|\int_{A} f_{k}\right\| & \leq \varepsilon+\left\|\sum_{i=1}^{m} \sum_{j=1}^{n} f_{k}\left(t_{i}\right) \ell\left(I_{i} \cap J_{j}\right)\right\|=\varepsilon+\left\|\sum_{i=3}^{m} f_{k}\left(t_{i}\right) \ell\left(I_{i} \cap A\right)\right\| \\
& \leq \varepsilon+\sup _{3 \leq i \leq m}\left\|f_{k}\left(t_{i}\right)\right\| \ell(I),
\end{aligned}
$$

and $k$ can be taken large enough so the last term is less than $\varepsilon$. Since $A$ is arbitrary, it follows that $\left\|f_{k}\right\|_{1}^{\prime} \leq 2 \varepsilon$ for large $k$.

It has been previously observed that $f$ is integrable and uniform integrability of a pointwise convergent sequence $\left\{f_{k}\right\}$ implies that $\lim \int_{\mathbb{R}} f_{k}=\int_{\mathbb{R}} f$ ([7], $[3,4,5])$. Since \|\|$_{1}$ and \|\|$_{1}^{\prime}$ are equivalent, the conclusion of Theorem 4 implies that $\lim \int_{A} f_{k}=\int_{A} f$ uniformly for $A \in \mathcal{A}$ giving a significant improvement particularly in the vector-valued result for the McShane integral. 
In [10] we established a convergence theorem for vector-valued McShane integrable functions which easily implies the Monotone Convergence (Beppo Levi) Theorem for scalar-valued functions. We now show that the conclusion of this generalized Monotone Convergence Theorem can be improved from \|\|$_{1}$-convergence to uniform integrability.

Since we are working over $\mathbb{R}$ instead of a bounded interval we need a preliminary lemma.

Lemma 5. There exists a positive McShane integrable function $\varphi: \mathbb{R} \rightarrow(0, \infty)$ and a gauge $\gamma\left(=\gamma_{\varphi}\right)$ such that $0 \leq S(\varphi, \mathcal{D}) \leq 1$ whenever $\mathcal{D}$ is a $\gamma$-fine partial tagged partition.

Proof. Let $\varphi$ be positive with $\int_{\mathbb{R}} \varphi=\frac{1}{2}$. Let $\gamma$ be a gauge with $\left|\frac{1}{2}-S(\varphi, \mathcal{D})\right|<$ $\frac{1}{2}$ whenever $\mathcal{D}$ is a $\gamma$-fine tagged partition of $\mathbb{R}$. Since $\varphi$ is positive, the result follows immediately from Henstock's Lemma.

Theorem 6. Let $f_{k} \subset M(\mathbb{R}, X)$ and suppose $\sum_{k=1}^{\infty} f_{k}=f$ pointwise with $\sum_{k=1}^{\infty}\left\|f_{k}\right\|_{1}<\infty$. If $F_{n}=\sum_{k=1}^{n} f_{k}$, then (i) $\left\{F_{n}\right\}$ is uniformly integrable, (ii) $f$ is integrable and (iii) $\left\|F_{n}-f\right\|_{1} \rightarrow 0$.

Proof. Let $\varepsilon>0$. For each $n$ pick a gauge $\gamma_{n}$ with $\gamma_{n}(z)$ bounded for every $z \in \mathbb{R}$ such that $\left\|\int_{\mathbb{R}} F_{n}-S\left(F_{n}, \mathcal{D}\right)\right\|<\varepsilon / 2^{n}$ whenever $\mathcal{D}$ is $\gamma_{n}$-fine. Pick $n_{0}$ such that $\sum_{k=n_{0}}^{\infty}\left\|f_{k}\right\|<\varepsilon$, and for every $t \in \mathbb{R}$ pick $n(t) \geq n_{0}$ such that $k \geq j \geq n(t)$ implies $\left\|\sum_{i=j}^{k} f_{i}(t)\right\|<\varepsilon \varphi(t)$, where $\varphi$ and $\gamma_{\varphi}$ are as in Lemma 5 . Define a gauge $\gamma$ on $\mathbb{R}$ by $\gamma(t)=\left(\bigcap_{j=1}^{n(t)} \gamma_{j}(t)\right) \cap \gamma_{\varphi}(t)$ for $t \in \mathbb{R}$ and $\gamma( \pm \infty)=\left(\bigcap_{j=1}^{n_{0}} \gamma_{j}( \pm \infty)\right) \cap \gamma_{\varphi}( \pm \infty)$ and set $n( \pm \infty)=n_{0}$. Suppose $\mathcal{D}$ $=\left\{\left(I_{i}, t_{i}\right): 1 \leq i \leq m\right\}$ is $\gamma$-fine. To establish (i), first note that $\mathcal{D}$ is $\gamma_{i}$-fine for $i=1, \ldots, n_{0}$ implies that $\left\|\int_{\mathbb{R}} F_{i}-S\left(F_{i}, \mathcal{D}\right)\right\|<\varepsilon / 2^{i}<\varepsilon$.

So, now fix $n>n_{0}$. Set $d_{1}=\left\{i: 1 \leq i \leq m, n\left(t_{i}\right) \geq n\right\}$ and $d_{2}=\{i: 1 \leq$ $\left.i \leq m, n\left(t_{i}\right)<n\right\}$, and note $\mathcal{D}_{1}=\left\{\left(I_{i}, t_{i}\right): i \in d_{1}\right\}$ is $\gamma_{n}$-fine by the definition of $\gamma$. Set $I=\cup\left\{I_{i}: i \in d_{1}\right\}$. We have, using Henstock's Lemma, 


$$
\begin{aligned}
& \left\|\int_{\mathbb{R}} F_{n}-S\left(F_{n}, \mathcal{D}\right)\right\| \leq \\
& \leq\left\|\int_{I} F_{n}-S\left(F_{n}, \mathcal{D}_{1}\right)\right\|+\left\|\sum_{i \in d_{2}} \sum_{j=1}^{n}\left\{\int_{I_{i}} f_{j}-f_{j}\left(t_{i}\right) \ell\left(I_{i}\right)\right\}\right\| \\
& \leq \varepsilon / 2^{n}+\left\|\sum_{i \in d_{2}} \sum_{j=1}^{n\left(t_{i}\right)}\left\{\int_{I_{i}} f_{j}-f_{j}\left(t_{i}\right) \ell\left(I_{i}\right)\right\}\right\| \\
& \quad+\left\|\sum_{i \in d_{2}} \sum_{j=n\left(t_{i}\right)+1}^{n} \int_{I_{i}} f_{j}\right\|+\left\|\sum_{i \in d_{2}} \sum_{j=n\left(t_{i}\right)+1}^{n} f_{j}\left(t_{i}\right) \ell\left(I_{i}\right)\right\| \\
& <\varepsilon+T_{1}+T_{2}+T_{3},
\end{aligned}
$$

with obvious notation for the $T_{i}$.

First, we estimate $T_{3}$ :

$$
T_{3} \leq \sum_{i \in d_{2}}\left\|\sum_{j=n\left(t_{i}\right)+1}^{n} f_{j}\left(t_{i}\right)\right\| \ell\left(I_{i}\right) \leq \sum_{i \in d_{2}} \varepsilon \varphi\left(t_{i}\right) \ell\left(I_{i}\right)=\varepsilon S\left(\varphi, \mathcal{D}_{2}\right) \leq \varepsilon,
$$

where $\mathcal{D}_{2}=\left\{\left(I_{i}, t_{i}\right): i \in d_{2}\right\}$.

Next,

$$
\begin{aligned}
T_{2} & =\sup \left\{\left|<x^{\prime}, \sum_{i \in d_{2}} \sum_{j=n\left(t_{i}\right)+1}^{n} \int f_{i}>\right|:\left\|x^{\prime}\right\| \leq 1\right\} \\
& \leq \sup \left\{\sum_{i \in d_{2}} \sum_{j=n\left(t_{i}\right)+1}^{n}\left|x^{\prime} f_{j}\right|:\left\|x^{\prime}\right\| \leq 1\right\} \\
& \leq \sup \left\{\sum_{i \in d_{2}} \sum_{j=n_{0}}^{n} \int\left|x_{i} f_{j}\right|:\left\|x^{\prime}\right\| \leq 1\right\} \\
& \leq \sup \left\{\sum_{j=n_{0}}^{n} \int_{\mathbb{R}}\left|x^{\prime} f_{j}\right|:\left\|x^{\prime}\right\| \leq 1\right\} \leq \sum_{j=n_{0}}^{n}\left\|f_{k}\right\|_{1}<\varepsilon .
\end{aligned}
$$

For $T_{1}$, let $s=\max \left\{n\left(t_{i}\right): i \in d_{2}\right\}$. Then 


$$
\begin{aligned}
T_{1} & =\left\|\sum_{i \in d_{2}}\left\{\int_{I_{i}} F_{n\left(t_{i}\right)}-F_{n\left(t_{i}\right)}\left(t_{i}\right) \ell\left(I_{i}\right)\right\}\right\| \\
& =\left\|\sum_{k=1}^{s} \sum_{\substack{i \\
n\left(t_{i}\right)=k}}\left\{\int_{I_{i}} F_{n\left(t_{i}\right)}-F_{n\left(t_{i}\right)}\left(t_{i}\right) \ell\left(I_{i}\right)\right\}\right\| \\
& \leq \sum_{k=1}^{s}\left\|\sum_{\substack{i \\
n\left(t_{i}\right)=k}}\left\{\int_{I_{i}} F_{n\left(t_{i}\right)}-F_{n\left(t_{i}\right)}\left(t_{i}\right) \ell\left(I_{i}\right)\right\}\right\| \leq \sum_{k=1}^{s} \varepsilon / 2^{k}<\varepsilon,
\end{aligned}
$$

by Henstock's Lemma since $\left\{\left(I_{i}, t_{i}\right): n\left(t_{i}\right)=k\right\}$ is $\gamma_{k}$-fine.

From (1), it follows that $\left\|\int_{\mathbb{R}} F_{n}-S\left(F_{n}, \mathcal{D}\right)\right\|<4 \varepsilon$ as required, and (i) holds.

Conditions (ii) and (iii) now follow from Theorem 4.

For the McShane integral we have from Theorem 6 a version of the Monotone Convergence Theorem (MCT) for the McShane integral. The conclusion in part (i) strengthens the "usual" conclusions in the MCT (see for example $[3,10.10])$.

Corollary 7. (MCT). Let $f_{k}: \mathbb{R} \rightarrow \mathbb{R}$ be integrable for every $k \in \mathbb{N}$ and suppose that $f_{k}(t) \uparrow f(t)$ for every $t$. If $\sup \left\{\int_{\mathbb{R}} f_{k}: k \in \mathbb{N}\right\}<\infty$, then (i) $\left\{f_{k}\right\}$ is uniformly $M$-integrable, (ii) $f$ is integrable and (iii) $\int_{\mathbb{R}} f_{k} \uparrow \int_{\mathbb{R}} f$.

Proof. Set $g_{0}=0$ and $g_{k}=f_{k}-f_{k-1}$ for $k \geq 1$. Then $\sum_{k=1}^{n} g_{k}=f_{n} \rightarrow f$ pointwise and

$$
\sum_{k=1}^{\infty} \int_{\mathbb{R}}\left|g_{k}\right|=\lim _{n} \sum_{k=1}^{n} \int_{\mathbb{R}}\left(f_{k}-f_{k-1}\right)=\lim _{n} \int_{\mathbb{R}} f_{k}=\sup _{k} \int_{\mathbb{R}} f_{k}<\infty .
$$

Hence, Theorem 6 gives the result.

Similarly, it was noted by McLeod that the same improvement can be obtained for the Monotone Convergence Theorem for the Henstock-Kurzweil integral ([7, p. 98] ; a similar result is obtained by Gordon $([3,13.18])$, but his proof uses Lebesgue integration.

We can use the MCT of Corollary 7 to obtain a similar generalization of the Dominated Convergence Theorem (DCT) for the McShane integral. A 
sequence $f_{k}: \mathbb{R} \rightarrow X$ is said to be uniformly McShane or $M$-Cauchy if for every $\varepsilon>0$ there exists a gauge $\gamma$ and $N$ such that $\left\|S\left(f_{i}, \mathcal{D}\right)-S\left(f_{j}, \mathcal{D}\right)\right\|<\varepsilon$ for $i, j \geq N$ and $\mathcal{D} \gamma$-fine. As in Theorem 4 of [5], we have

Proposition 8. Let $f_{k}: \mathbb{R} \rightarrow X$ be integrable for every $k \in \mathbb{N}$. Then $\left\{f_{k}\right\}$ is uniformly $M$-Cauchy if and only if $\left\{f_{k}\right\}$ is uniformly integrable and $\lim \int_{\mathbb{R}} f_{k}$ exists.

Corollary 9. (DCT) Let $f_{k}: \mathbb{R} \rightarrow \mathbb{R}$ be integrable for every $k \in \mathbb{N}$ and suppose $f_{k} \rightarrow f$ pointwise. Assume there exists $g: \mathbb{R} \rightarrow \mathbb{R}$, integrable and such that $\left|f_{i}-f_{j}\right| \leq g$ for all $i, j$. Then (i) $\left\{f_{k}\right\}$ is uniformly integrable, (ii) $f$ is integrable and (iii) $\int_{\mathbb{R}}\left|f_{k}-f\right| \rightarrow 0$.

Proof. Set $t_{j k}=\vee\left\{\left|f_{m}-f_{n}\right|: j \leq m \leq n \leq k\right\}$. For each $j\left\{t_{j k}\right\}_{k}$ is increasing and converges to the function $t_{j}=\vee\left\{\left|f_{m}-f_{n}\right|: j \leq m \leq n\right\}$ with $0 \leq t_{j} \leq g$. Corollary 7 implies that $t_{j}$ is integrable and $\int_{\mathbb{R}} t_{j} \leq \int_{\mathbb{R}} g$. Now $0 \leq t_{j+1} \leq t_{j}$ and $t_{j} \rightarrow 0$ pointwise so Corollary 7 implies that $\int_{\mathbb{R}} t_{j} \downarrow 0$.

Let $\varepsilon>0$. There exists $N$ such that $\int_{\mathbb{R}} t_{N}<\varepsilon$, and there exists a gauge $\gamma$ such that $\left|\int_{\mathbb{R}} t_{N}-S\left(t_{N}, \mathcal{D}\right)\right|<\varepsilon$ when $\mathcal{D}$ is $\gamma$-fine. If $i, j \geq N$, we have $\left|S\left(f_{i}, \mathcal{D}\right)-S\left(f_{j}, \mathcal{D}\right)\right| \leq S\left(\left|f_{i}-f_{j}\right|, \mathcal{D}\right) \leq S\left(t_{N}, \mathcal{D}\right)<\int_{\mathbb{R}} t_{N}+\varepsilon<2 \varepsilon$ when $\mathcal{D}$ is $\gamma$-fine. Hence, $\left\{f_{i}\right\}$ is uniformly $M$-Cauchy. It follows from Proposition 8 that $\left\{f_{i}\right\}$ is uniformly integrable and (i) holds.

Conditions (ii) and (iii) follow from Theorem 6.

McLeod obtained a similar improvement in the DCT for the HenstockKurzweil integral ([7, p. 98] ); Gordon also obtained this result but employed the Lebesgue integral $([3,13.17])$.

Finally, in conclusion it should be noted that the results above are also valid with $\mathbb{R}$ being replaced by $\mathbb{R}^{n}$; only the notation becomes more cumbersome.

If $I=[a, b)$ is a bounded interval, it is straightforward to generalize the Henstock-Kurzweil integral to functions $f: I \rightarrow X$. If $H K(I, X)$ is the space of all $X$-valued Henstock-Kurzweil integrable functions defined on $I$, then $H K(I, X)$ has a natural semi-norm defined by $\|f\|=\sup \left\{\left\|\int_{a}^{t} f\right\|: a \leq t \leq b\right\}$ $([6,11.1])$.

Problem: Are there analogues of Theorems 4 and 6 for the HenstockKurzweil integral? 
The proofs of these results above are not valid for the Henstock-Kurzweil integral. In Theorem $4 \mathrm{McShane}$ tagged partitions were used and the proof of Theorem 6 used the absolute integrability of the scalar-valued McShane integral in estimating $T_{2}$ so different techniques would be required.

The referee has observed that the sequence $f_{k}(t)=(\sin t) / t$ for $1 \leq t \leq 2 k \pi$ and $f_{k}(t)=0$ for $t>2 k \pi$ gives a counter-example to Theorem 3 for the Henstock-Kurzweil integral.

\section{References}

[1] J. De Pree and C. Swartz, Introduction to Real Analysis, Wiley, N.Y., 1987.

[2] D. Fremlin and J. Mendoza, "On the Integration of Vector-Valued Functions," Illinois J. Math., 38 (1994), 127-147.

[3] R. Gordon, The Integrals of Lebesgue, Denjoy, Perron and Henstock, Amer. Math. Soc., Providence, 1994.

[4] R. Gordon, "The McShane Integral of Banach-Valued Functions," Illinois J. Math, 34 (1990), 557-567.

[5] R. Gordon, "Another Look at a Convergence Theorem for the Henstock Integral," Real Anal. Exch., 15 (1989/90), 724-728.

[6] Lee Peng Yee, Lanzhou Lectures on Henstock Integration, World Sci. Publ., Singapore, 1989.

[7] R. McLeod, The Generalized Riemann Integral, Math. Assoc. Amer., Providence, 1980.

[8] E. J. McShane, Unified Integration, Academic Press, N.Y., 1983.

[9] C. Swartz, Measure, Integration and Function Spaces, World Sci. Publ. Singapore, 1994.

[10] C. Swartz, "Beppo Levi's Theorem for the Vector-Valued McShane Integral and Applications," Bull. Belgian Math. Soc., to appear. 